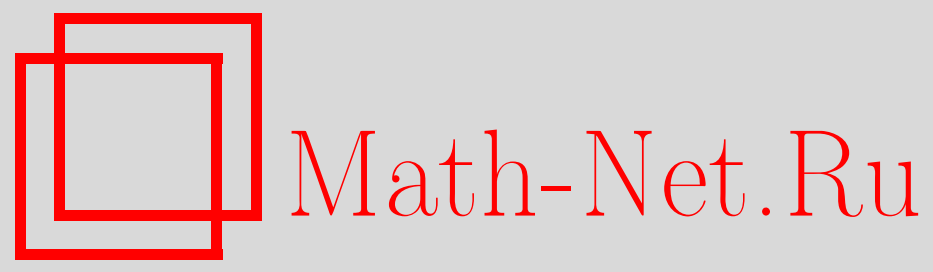

Д. А. Куропаткин, Вероятностный алгоритм нахождения граничного ранга неотрицательной матрицы, Дискрет. матем., 2005, том 17, выпуск 1, 147-156

DOI: https://doi.org/10.4213/dm94

Использование Общероссийского математического портала Math-Net.Ru подразумевает, что вы прочитали и согласны с пользовательским соглашением http: //www . mathnet.ru/rus/agreement

Параметры загрузки:

IP: 54.157 .27 .8

26 апреля 2023 г., 08:44:06 
УдК 519.1

\title{
Вероятностный алгоритм нахождения граничного ранга неотрицательной матрицы
}

\author{
() 2005 г. Д. А. Куропаткин
}

\begin{abstract}
Предложен вероятностный алгоритм нахождения граничного ранга матрицы, элементы которой неотрицательны. Приведена оценка сложности данного алгоритма и получена оценка сверху для вероятности неправильного нахождения искомого параметра.
\end{abstract}

\section{1. Введение}

В монографии [1] отмечается, что одной из основных глобальных комбинаторных характеристик матрицы является ее граничный ранг. Параграф 3 главы 2 в [1] посвящен оцениванию величина граничного ранга матриц с неотрицательными элементами.

В данной работе предлагается вероятностный алгоритм, с помощью которого находится граничный ранг матрицы с неотрицательным элементами. Во втором параграфе вводятся основные определения. В третьем параграфе описывается предлагаемый алгоритм и приводится оценка сложности его реализации. В четвертом параграфе содержатся необходимые доказательства.

По сути в предлагаемом алгоритме вычисление граничного ранга заменяется на неоднократное вычисление ранга некоторой матрицы, похожей на исходную. Данный подход основан на известной идее (см., например, [2]) нахождения перманента конкретной матрицы через определитель некоторой другой матрицы. Напомним, что в общем случае было показано, что перманенты матриц нельзя обратить в детерминанты посредством линейного преобразования.

\section{2. Основные определения}

Будем придерживаться обозначений и определений монографии [1]. Скажем, что матрица $A$ имеет размер $m \times n$, если у нее $m$ строк и $n$ столбцов. Везде далее будем считать, что $m \leqslant n$. Матрицу, элементами которой являются неотрицательные числа, будем называть неотрицательной. Совокупность всех неотрицательных матриц размера $m \times n$ обозначим через $\mathscr{R}_{m, n}^{+}$или $\mathscr{R}_{n}^{+}$при $m=n$. Совокупность всех матриц размера $m \times n$ над полем действительный чисел будем обозначать через $\mathscr{R}_{m, n}$ или $\mathscr{R}_{n}$ при $m=n$. Множество натуральных чисел обозначим через $\mathbf{N}$, и пусть $N_{s}^{k}-$ множество векторов длины $k$, элементами которых являются натуральные числа от 1 до $s$. 
Пусть $A=\left(a_{i, j}\right)$ - неотрицательная матрица размера $m \times n$. Ее носителем назовем $(0,1)$-матрицу $\left(a_{i, j}^{\prime}\right)$ размера $m \times n$, в которой

$$
a_{i, j}^{\prime}= \begin{cases}1, & \text { если } a_{i, j} \neq 0 \\ 0, & \text { если } a_{i, j}=0\end{cases}
$$

Определение 1. Граничным рангом матрицы $A$ называется наибольшее число ее ненулевых элементов, никакие два из которых не лежат в одной строке или одном столбце.

Граничный ранг матрицы $A$ обозначается $\rho(A)$. Два элемента матрицы называются коллинеарными, если они лежат либо в одной строке, либо в одном столбце. Диагональю в матрице размера $m \times n$ называется совокупность из $\min (m, n)$ ее попарно неколлинеарных элементов. Диагональ, состоящая из ненулевых элементов, называется ненулевой. Будем говорить о частичной диагонали в матрице размера $m \times n$, подразумевая под этим набор из ненулевых попарно неколлинеарных элементов матрицы. Иногда будем говорить просто о диагонали матрицы, имея в виду ненулевую диагональ. Из контекста будет ясно, о какой диагонали идет речь.

Перманентом $\operatorname{per}(A)$ матрицы $A=\left(a_{i, j}\right) \in \mathscr{R}_{m, n}$ называется число

$$
\operatorname{per}(A)=\sum_{\sigma} a_{1 \sigma(1)} a_{2 \sigma(2)} \ldots a_{m \sigma(m)},
$$

где суммирование ведется по всем упорядоченным наборам $\sigma=(\sigma(1), \ldots, \sigma(m))$ объема $m$ из множества $\{1, \ldots, n\}$.

Определение 2. Граничным рангом матрицы $A$ называется наибольший размер квадратной подматрицы, имеющей ненулевой перманент.

Ясно, что два определения граничного ранга матрицы эквивалентны.

\section{3. Алгоритм нахождения граничного ранга}

Пусть $A=\left(a_{i, j}\right)$ - матрица из $\mathscr{R}_{m, n}^{+}$, число ненулевых элементов которой равно $k$. В данном параграфе мы опишем вероятностный алгоритм нахождения граничного ранга $\rho(A)$.

Пусть

$$
L(A)=\left\{(i, j), 1 \leqslant i \leqslant m, 1 \leqslant j \leqslant n: a_{i, j} \neq 0\right\},
$$

а $x$ - вектор из $k$ переменных $\left(x_{i, j}\right)$, где индексы $(i, j) \in L(A)$. Кольцо многочленов от $k$ переменных $\left(x_{i_{n} j}\right)$ над полем действительных чисел обозначим $\mathbf{R}[x]$. Далее кольцо матриц размера $m \times n$ над указанным кольцом многочленов обозначаем через $\mathbf{R}[x]_{m, n}$.

Матрицу $B_{A}(x)$ из $\mathbf{R}[x]_{m, n}$ назовем сопутствующей для матрицы $A$, если элемент матрищы $B_{A}(x)$, расположенный в $i$-й строке и $j$-м столбце, равен $x_{i, j}$ при $a_{i, j}>0$ и равен 0 при $a_{i, j}=0$. Здесь, как и в предыдущем абзаце, символом $x$ обозначается вектор из $k$ переменных $x_{i, j}$. Ясно, что носители матриц $A$ и $B_{A}(x)$ совпадают. Для произвольного вектора $d \in N_{s}^{k}$ определим матрицу $B_{A}(d)$ естественным образом: вместо компонент вектора $x$ построчно, начиная с начала первой строки, расставляются последовательно, начиная с первой, компоненты вектора $d$. Произвольный вектор $d \in N_{s}^{k}$ называем корнем многочлена $\operatorname{det} B_{A}(x)$ в случае, если $\operatorname{det} B_{A}(d)=0$. 
В описании и работе алгоритма участвуют два параметра, $l$ и $s$. Они являются входными параметрами, и их значения задаются до начала работы алгоритма. Смысл их будет ясен из описания алгоритма. Здесь скажем лишь, что значения этих параметров влияют на вероятность правильного нахождения граничного ранга матрицы при работе алгоритма. Из дальнейших утверждений будет ясно, что чем больше значения данных параметров, тем больше вероятность того, что алгоритм правильно определит граничный ранг матрицы.

Алгоритм. (1) Присваиваем переменной $i$ значение 1 .

(2) Случайно равновероятно выбираем вектор $d(i) \in N_{s}^{k}$.

(3) Находим ранг матрицы $B_{A}(d(i))$. Это можно сделать, например, используя прямой ход алгоритма Гаусса - приведение матрицы $B_{A}(d(i))$ к ступенчатому виду (описание данного алгоритма можно найти, например, в [3]). Пусть полученный ранг равен $r(i)$.

(4) Если значение переменной $i$ меньше значения параметра $l$, то увеличиваем значение $i$ на единицу и переходим к пункту 2 . Если $i=l$, то находим величину

$$
\rho^{\prime}(A)=\max \{r(1), \ldots, r(l)\}
$$

и объявляем $\rho^{\prime}(A)$ искомым значением $\rho(A)$.

В дальнейшем будем говорить, что произошла ошибка при работе Алгоритма, если для матрицы $A$, которая подается на вход алгоритма, не выполняется равенство

$$
\rho^{\prime}(A)=\rho(A) \text {. }
$$

Предложение 1. Пусть матрича А принадлежит $\mathscr{R}_{m, n}^{+} u s>2$. Тогда

(1) $\rho^{\prime}(A) \leqslant \rho(A)$;

(2) вероятность ошибки при работе Алгоритма $\left(\rho^{\prime}(A) \neq \rho(A)\right)$ оченивается следующим образом:

$$
\mathbf{P}\left(\rho^{\prime}(A) \neq \rho(A)\right) \leqslant\left(\frac{2}{s}\right)^{l} .
$$

Предложение 2. Для любой матрицы А из множества $\mathscr{R}_{m, n}^{+}$число сложений и умножений рачиональных чисел, которые выполняет Алгоритм, есть $O\left(\mathrm{~nm}^{2}\right)$.

\section{4. Доказательства}

Пусть $A$ - матрица из $\mathscr{R}_{n}$ с $k$ ненулевыми элементами и пусть $a_{1,1} \neq 0$. Обозначим через $F$ матрицу, полученную из $A$ вычеркиванием первой строки и первого столбца, а через $G$ матрицу, полученную из $A$ заменой элемента, расположенного на месте $(1,1)$ нулем. Для любого действительного вектора $d=\left(d_{i, j}\right),(i, j) \in L(A)$, обозначим через $d_{1}$ вектор $\left(d_{i, j}\right)$, где $(i, j) \in L(A)$ и либо $i \neq 1$, либо $j \neq 1$, а через $d_{2}$ такой вектор $\left(d_{i, j}\right)$, где $(i, j) \in L(A)$ и $(i, j) \neq(1,1)$. Таким образом, векторы $d_{1}$ и $d_{2}$ являются частями вектора $d$. 
Лемма 1. Пусть $A$ - матрича из $\mathscr{R}_{n} c k$ ненулевыми элементами, удовлетворяющая следующему условию: существует вектор $d^{*}=\left(d_{i, j}^{*}\right),(i, j) \in L(A)$, длины $k$ с действительными элементами такой, что $\operatorname{det} B_{A}\left(d^{*}\right) \neq 0$.

Тогда существует вектор $d=\left(d_{i, j}\right),(i, j) \in L(A)$, принадлежаший множеству $N_{s}^{k}$, $s>1$, такой, что

$$
\operatorname{det} B_{A}(d) \neq 0
$$

Доказательство. Доказательство проведем индукцией по числу $k$ ненулевых элементов матрицы $A$.

В случае $k=2$ утверждение леммы очевидно. Действительно, в этом случае, в силу условия леммы, матрица $A$ должна иметь размер, не больший $2 \times 2$, и два ненулевых элемента должны быть расположены либо на местах $(1,1)$ и $(2,2)$, либо на местах $(1,2)$ и $(2,1)$. Тогда, очевидно, что можно подобрать вектор $d$ из $N_{s}^{k}, s>1$, такой, что $\operatorname{det} B_{A}(d) \neq 0$.

Пусть утверждение леммы верно для любой матрицы $A$, имеющей ровно $k-1$ ненулевых элементов. Рассмотрим случай, когда матрица $A$ имеет ровно $k$ ненулевых элементов. Из условия леммы следует, что в первой строке матрицы $B_{A}(x)$ имеется хотя бы один ненулевой элемент. Без ограничения общности будем считать, что на месте $(1,1)$ расположен ненулевой элемент $x_{1,1}$. Очевидно, что для любого действительного вектора $d=\left(d_{i, j}\right),(i, j) \in L(A)$, верно представление

$$
\operatorname{det} B_{A}(d)=d_{1,1} \operatorname{det} B_{F}\left(d_{1}\right)+\operatorname{det} B_{G}\left(d_{2}\right) .
$$

Напомним, что $F, G, d_{1}$ и $d_{2}$ определены в этом параграфе перед формулировкой леммы 1 .

Так как существует действительный вектор $d^{*}$ длины $k$ такой, что $\operatorname{det} B_{A}\left(d^{*}\right) \neq 0$, равенства $\operatorname{det} B_{F}\left(d_{1}^{*}\right)=0$ и $\operatorname{det} B_{G}\left(d_{2}^{*}\right)=0$ не могут выполняться одновременно.

Пусть без ограничения общности $\operatorname{det} B_{F}\left(d_{1}^{*}\right) \neq 0$. По предположению индукции в множестве $N_{s}^{k}$ существует вектор $c=\left(c_{i, j}\right),(i, j) \in L(F)$, такой, что $\operatorname{det} B_{F}(c) \neq 0$. Тогда рассмотрим произвольный вектор $d_{2}=\left(d_{i, j}\right),(i, j) \in L(G)$, следующего вида: при $j \neq 1$, $(1, j) \in L(A)$ элемент $d_{1, j}$ является произвольным числом из множества $N_{s}^{1}$, при $i \neq 1$, $(i, 1) \in L(A)$, элемент $d_{i, 1}$ также является произвольным числом из множества $N_{s}^{1}$, и $d_{i, j}=c_{i-1, j-1}$, если $i>1, j>1,(i, j) \in L(A)$. Таким образом, рассматривается вектор $d_{2}$, соответствующие компоненты которого совпадают с компонентами вектора $c$. Ясно, что для описанного вектора $d_{2}$ существует $d_{1,1} \in N_{s}^{1}$, при котором $\operatorname{det} B_{A}(d) \neq 0$, где $d=\left(d_{i, j}\right),(i, j) \in L(A)$. Случай $\operatorname{det} B_{G}\left(d_{2}^{*}\right) \neq 0$ рассматривается аналогично.

Лемма доказана.

Лемма 2. Пусть $s>2$, матрииа $A$ принадлежит $\mathscr{R}_{n}$, имеет $k$ ненулевых элементов $и$ удовлетворяет следуюшему условию: существует вектор $d^{*}=\left(d_{i, j}^{*}\right),(i, j) \in L(A), c$ действительными элементами такой, что

$$
\operatorname{det} B_{A}\left(d^{*}\right) \neq 0
$$

Тогда число $X_{A}$ векторов $d=\left(d_{i, j}\right)$, где $(i, j) \in L(A)$, принадлежащих множеству $N_{s}^{k} u$ таких, что

$$
\operatorname{det} B_{A}(d)=0
$$

допускает оченку

$$
X_{A} \leqslant 2 s^{k-1}
$$


Доказательство. Доказательство проведем индукцией по числу ненулевых элементов матрицы $A$.

В случае $k=2$ утверждение леммы очевидно. Действительно, в этом случае, в силу условий леммы, матрица $A$ должна иметь размер, не больший $2 \times 2$, и два ненулевых элеменга должны быть расположены либо на местах $(1,1)$ и $(2,2)$, либо на местах $(1,2)$ и $(2,1)$. Ясно, что в обоих случаях при любом натуральном $s$ многочлен $\operatorname{det} B_{A}(x)$ не имеет корней, принадлежащих множеству $N_{s}^{2}$.

Пусть утверждение леммы верно для любой матрицы $A$, имеющей ровно $k-1$ ненулевых элементов. Рассмотрим случай матрицы $A$, имеющей ровно $k$ ненулевых элементов. Среди всевозможных векторов $d=\left(d_{i, j}\right),(i, j) \in L(A)$, принадлежащих множеству $N_{s}^{k}$, оценим число таких векторов, что $\operatorname{det} B_{A}(d)=0$. Как и в лемме 1 , считаем, без ограничения общности, что в матрице $B_{A}(x)$ на месте $(1,1)$ расположен ненулевой элемент $x_{1,1}$. Ясно, что при любом векторе $d=\left(d_{i, j}\right),(i, j) \in L(A)$, верно соотношение (1) из доказательства леммы 1.

Положим $x_{1}=\left(x_{i, j}\right)$, где $(i, j) \in L(A)$ и либо $i \neq 1$, либо $j \neq 1$, а $x_{2}=\left(x_{i, j}\right)$, где $(i, j) \in L(A),(i, j) \neq(1,1)$. Рассмотрим три возможных случая,

(1) $\operatorname{det} B_{F}\left(x_{1}\right) \equiv 0$,

(2) $\operatorname{det} B_{G}\left(x_{2}\right) \equiv 0$, и

(3) $\operatorname{det} B_{F}\left(x_{1}\right) \not \equiv 0$ и $\operatorname{det} B_{G}\left(x_{2}\right) \not \equiv 0$.

Напомним, что матрицы $F$ и $G$ определены в этом параграфе перед формулировкой леммы 1.

1. Пусть

$$
\operatorname{det} B_{F}\left(x_{1}\right) \equiv 0 \text {. }
$$

Из (1) ясно, что матрица $G$ удовлетворяет условию леммы и содержит $k-1$ ненулевых элементов. По предположению индукции, число корней уравнения

$$
\operatorname{det} B_{G}\left(x_{2}\right)=0
$$

не превосходит $2 s^{k-2}$. Отсюда для произвольного $d_{1,1}$ из $N_{s}^{1}$ и вектора $d_{2}$ из множества корней многочлена $\operatorname{det} B_{G}\left(x_{2}\right)$ справедливо равенство

$$
\operatorname{det} B_{A}\left(d_{1,1}, d_{2}\right)=0 .
$$

С учетом (1) в данном случае $X_{A}$ можно оценить величиной $2 s^{k-2} s$. Итак, в рассматриваемой ситуации шаг индукщии оправдан.

2. Случай, когда многочлен $\operatorname{det} B_{G}\left(x_{2}\right)$ тождественно равен нулю, рассматривается аналогично.

3. Осталось рассмотреть возможность

$$
\operatorname{det} B_{F}\left(x_{1}\right) \not \equiv 0, \quad \operatorname{det} B_{G}\left(x_{2}\right) \not \equiv 0 \text {. }
$$

Пусть $l_{1}$ - число переменных в первой строке матрицы $B_{A}(x)$, за исключением переменной $x_{1,1}$, а $l_{2}$ - число переменных в первом столбце матрицы $B_{A}(x)$, за исключением переменной $x_{1,1}$. Если $l_{1}=0$ или $l_{2}=0$, то для любого действительного вектора $d_{2}^{\prime}$ 
определитель матрицы $B_{G}\left(d_{2}^{\prime}\right)$ в правой части равенства (1) тождественно равен нулю, что для рассматриваемого случая невозможно.

Таким образом, в дальнейшем считаем, что $\operatorname{det} B_{F}\left(x_{1}\right)$ и $\operatorname{det} B_{G}\left(x_{2}\right)$ не равны тождественно нулю и $l_{1} l_{2}>0$.

Пусть $C$ - множество векторов $d_{2}=\left(d_{i, j}\right),(i, j) \in L(A)$ и $(i, j) \neq(1,1)$, принадлежащих множеству $N_{s}^{k-1}$. Обозначим через $C_{1}$ множество векторов $d_{2}$ из множества $C$, для которых $\operatorname{det} B_{F}\left(d_{1}\right)=0$. Здесь $d_{1}$ - часть вектора $d_{2}$, равная $\left(d_{i, j}\right)$, где $(i, j) \in L(A)$ и $i \neq 1, j \neq 1$. Через $C_{2}$ обозначим множество векторов $d_{2}$ из множества $C$, для которых выполняется равенство $\operatorname{det} B_{G}\left(d_{2}\right)=0$.

Оценим число $X_{A}$ корней многочлена $\operatorname{det} B_{A}(x)$, принадлежащих множеству $N_{s}^{k}$. Для этого разобьем множество возможных векторов $d=\left(d_{i, j}\right),(i, j) \in L(A)$, содержащихся в $N_{s}^{k}$, на четыре непересекающихся подмножества и оценим число корней, принадлежащих каждому из этих подмножеств.

a) Рассмотрим векторы $d \in N_{s}^{k}$, для которых

$$
\operatorname{det} B_{F}\left(d_{1}\right) \operatorname{det} B_{G}\left(d_{2}\right) \neq 0 \text {. }
$$

Число подвекторов $d_{2}$ таких, что $\operatorname{det} B_{F}\left(d_{1}\right) \operatorname{det} B_{G}\left(d_{2}\right) \neq 0$ равно $s^{k-1}-\left|C_{1} \cup C_{2}\right|$, здесь $d_{1}=\left(d_{i, j}\right),(i, j) \in L(A), i \neq 1, j \neq 1$ - часть вектора $d_{2}$. Из (1) ясно, что для каждого указанного вектора $d_{2}$ существует единственное действительное число $d_{1,1}$ такое, что $\operatorname{det} B_{A}\left(d_{1,1}, \dot{d}_{2}\right)=0$. Хотя число $d_{1.1}$ не обязано принадлежать множеству $N_{s}^{1}$, оценим сверху число векторов $d$ из $N_{s}^{k}$, для которых $\operatorname{det} B_{F}\left(d_{1}\right) \operatorname{det} B_{G}\left(d_{2}\right) \neq 0$, а $\operatorname{det} B_{A}\left(d_{1,1}, d_{2}\right)=0$, величиной $s^{k-1}-\left|C_{1} \cup C_{2}\right|$.

б) Рассмотрим подмножество векторов $d$ из $N_{s}^{k}$, для которых

$$
\operatorname{det} B_{F}\left(d_{1}\right)=0, \quad \operatorname{det} B_{G}\left(d_{2}\right) \neq 0 .
$$

Из (1) следует, что для векторов $d_{2}$, которые являются частями указанных векторов $d$, не существует действительного числа $d_{1,1}$ такого, что $\operatorname{det} B_{A}\left(d_{1,1}, d_{2}\right)=0$. Таким образом, число корней в рассматриваемом подмножестве равно нулю.

в) Аналогично предыдущему случаю, пусто множество векторов $d$ из $N_{s}^{k}$, для которых

$$
\operatorname{det} B_{F}\left(d_{1}\right) \neq 0, \quad \operatorname{det} B_{G}\left(d_{2}\right)=0, \quad \operatorname{det} B_{A}(d)=0 .
$$

г) Наконец, рассмотрим подмножество векторов $d$ из $N_{s}^{k}$, для которых справедливы равенства

$$
\operatorname{det} B_{F}\left(d_{1}\right)=0, \quad \operatorname{det} B_{G}\left(d_{2}\right)=0 .
$$

Число векторов $d_{2}$, являющихся частями указанных векторов $d$, для которых выполняются равенства $\operatorname{det} B_{F}\left(d_{1}\right)=0$ и $\operatorname{det} B_{G}\left(d_{2}\right)=0$ (вектор $d_{1}$ имеет тот же смысл, что и в пункте а)), равно $\left|C_{1} \cap C_{2}\right|$. В данном случае, для произвольного числа $d_{1,1}$ из $N_{s}^{1}$, справедливо равенство $\operatorname{det} B_{A}\left(d_{1,1}, d_{2}\right)=0$. Значит, в рассматриваемом случае, число корней многочлена $\operatorname{det} B_{A}(x)$ равно $s\left|C_{1} \cap C_{2}\right|$.

Итак, число $X_{A}$ корней из множества $N_{s}^{k}$ многочлена $\operatorname{det} B_{A}(x)$ оценивается сверху числом

$$
s^{k-1}-\left|C_{1} \cup C_{2}\right|+s t\left|C_{1} \cap C_{2}\right| \text {. }
$$

Следовательно, справедлива оценка

$$
X_{A} \leqslant s^{k-1}+(s-1)\left|C_{1} \cap C_{2}\right| .
$$


Оценим мощность множества $C_{1} \cap C_{2}$. Нам необходимо оценить число векторов $d_{2}=\left(d_{i, j}\right)$, где $(i, j) \in L(A),(i, j) \neq(1,1)$, принадлежащих множеству $N_{s}^{k-1}$, при подстановке которых многочлены $\operatorname{det} B_{F}\left(x_{1}\right)$ и $\operatorname{det} B_{G}\left(x_{2}\right)$ одновременно обращаются в нуль. Матрица $F$ удовлетворяет условию леммы и содержит ровно $k-1-l_{1}-l_{2}$ ненулевых элементов. По предположению индукщии, число корней уравнения $\operatorname{det} B_{F}\left(x_{1}\right)=0$ из множества $N_{s}^{k-1-l_{1}-l_{2}}$ оценивается сверху величиной $2 s^{k-1-l_{1}-l_{2}}$. Данное число является оценкой числа векторов $d_{1}=\left(d_{i, j}\right)$, где $(i, j) \in L(A)$ и либо $i \neq 1$, либо $j \neq 1$, которые являются частями векторов $d_{2}$ и при подстановке которых многочлен $\operatorname{det} B_{F}\left(x_{1}\right)$ обращается в нуль. Ясно, что при любых значениях чисел $\left(d_{1, j}\right)$ и $\left(d_{i, 1}\right)$, где $(i, j) \in L(A)$ и $(i, j) \neq(1,1)$ (всего $l_{1}+l_{2}$ чисел) значение многочлена $\operatorname{det} B_{F}\left(x_{1}\right)$ останется равным нулю.

Для произвольного вектора $d_{1}$, обращающего в нуль $\operatorname{det} B_{F}\left(x_{1}\right)$, среди всевозможных вариантов чисел $\left(d_{1, j}\right)$ и $\left(d_{i, 1}\right)$, где $(i, j) \in L(A)$ и $(i, j) \neq(1,1)$, оценим число тех, для которых общий вектор $d_{2}$ обращает в нуль и многочлен $\operatorname{det} B_{G}\left(x_{2}\right)$.

Пусть $d^{(1)}=\left(d_{1, j}\right)$, где $(1, j) \in L(A), j \neq 1$ и и $d^{(2)}=\left(d_{i, 1}\right)$, где $(i, 1) \in L(A), i \neq 1$. Ясно, что вектор $d^{(1)}$ имеет длину $l_{1}$, а вектор $d^{(2)}$ имеет длину $l_{2}$.

Пусть $D-$ множество векторов $d^{(2)}$ таких, что при любом значении вектора $d^{(1)}$ общий вектор $d_{2}$ (состоящий из $d_{1}, d^{(1)}$ и $d^{(2)}$ ) обращает в нуль многочлен $\operatorname{det} B_{G}\left(x_{2}\right)$. В этих обозначениях существует $|D| s^{l_{1}}$ векторов $\left(d^{(1)}\right.$ и $\left.d^{(2)}\right)$ длины $l_{1}+l_{2}$, каждый из которых вместе с $d_{1}$ обращает в нуль $\operatorname{det} B_{G}\left(x_{2}\right)$.

Если вектор $d^{(2)}$ не принадлежит $D$, то после замены в $\operatorname{det} B_{G}\left(x_{2}\right)$ соответствующей части вектора $x_{2}$ на вектор, составленный из $d^{(2)}$ и $d_{1}$, получается ненулевой многочлен $f\left(x_{2}\right)$. Данный многочлен представляет собой линейную комбинацию переменных первой строки матрищы $B_{G}\left(x_{2}\right)$. Тогда число векторов $d^{(1)}$, при которых общий вектор $d^{(2)}, d^{(1)}$ и $d_{1}$ обращает в нуль $\operatorname{det} B_{G}\left(x_{2}\right)$, очевидно, не превосходит числа $s^{l_{1}-1}$. Действительно, выбираем произвольную переменную из $f\left(x_{2}\right)$ (по предположению, хотя бы одна такая переменная найдется). Остальные $l_{1}-1$ переменные первой строки матрицы $B_{G}\left(x_{2}\right)$ заменяем на произвольные значения из множества $1, \ldots, s$. Ясно, что существует не более одного значения выбранной переменной, при котором соответствующий определитель обращается в нуль. Таким образом, в данном случае число искомых векторов (векторов $d^{(1)}$ и $d^{(2)}$, которые вместе с $d_{1}$ обращают в нуль $\left.\operatorname{det} B_{G}\left(x_{2}\right)\right)$, не превосходит $\left(s^{l_{2}}-|D|\right) s^{l_{1}-1}$.

Итак, мы получили промежуточную оценку

$$
\left|C_{1} \cap C_{2}\right| \leqslant 2 s^{k-2-l_{1}-l_{2}}\left(|D| s^{l_{1}}+\left(s^{l_{2}}-|D|\right) s^{l_{1}-1}\right) .
$$

Осталось оценить мощность множества $D$. Рассмотрим матрицу $B_{G}\left(x_{2}\right)$, когда в ней соответствующие компоненты вектора $x_{2}$ заменены на значения $d_{1}$ и $d^{(2)}$. Ясно, что вектор $d^{(2)}$ принадлежит $D$ тогда и только тогда, когда в рассматриваемой матрице строки за исключением первой линейно зависимы с действительными коэффищиентами. Так как $\operatorname{det} B_{F}\left(d_{1}\right)=0$, строки матрицы $B_{F}\left(d_{1}\right)$ линейно зависимы. Вспомним, что строки рассматриваемой матрицы, за исключением первых элементов, совпадают со строками матрищы $B_{F}\left(d_{1}\right)$. Следовательно, для того чтобы вектор $d^{(2)}$ принадлежал $D$ необходимо, чтобы компоненты вектора $d^{(2)}$ были линейно зависимы с заданными (из соотношения $\operatorname{det} B_{F}\left(d_{1}\right)=0$ ) коэффициентами. Число векторов с таким условием оценивается сверху числом $s^{l_{2}-1}$ (как и в случае, рассмотренном двумя абзацами раньше, все, кроме одной, компоненты могут быть произвольными). Итак,

$$
\begin{aligned}
\left|C_{1} \cap C_{2}\right| & \leqslant 2 s^{k-2-l_{1}-l_{2}}\left(s^{l_{2}-1} s^{l_{1}}+\left(s^{l_{2}}-s^{l_{2}-1}\right) s^{l_{1}-1}\right) \\
& =4 s^{k-3}-2 s^{k-4}
\end{aligned}
$$


Следовательно,

$$
\begin{aligned}
X_{A} & \leqslant s^{k-1}+(s-1) 4 s^{k-3}-2 s^{k-4} \\
& =s^{k-1}+4 s^{k-2}-4 s^{k-3}-2 s^{k-4} .
\end{aligned}
$$

Очевидно, что

$$
4 s^{k-2}-4 s^{k-3}-2 s^{k-4} \leqslant s^{k-1}
$$

при $s \geqslant 3$.

Таким образом, лемма доказана полностью.

Замечание 1. Экспериментальные данные показывают, что при малых значениях $k$ и $n$ число $X_{A}$ корней меньше, чем $s^{k-1}$, и при росте $k$ и $n$ разница между приведенной в лемме оценкой и истинным значением значительно растет.

Лемма 3. Пусть $s>1$, матрича А принадлежит $\Re_{m, n}^{+}$и имеет $k$ ненулевых элементов. Тогда справедливо равенство

$$
\rho(A)=\max _{d \in N_{s}^{k}} \operatorname{rank}\left[B_{A}(d)\right]
$$

здесь $\operatorname{rank}\left[B_{A}(d)\right]-$ ранг матричы $B_{A}(d)$.

Доказательство. При доказательстве будем пользоваться следующими определениями ранга и граничного ранга матрицы.

Напомним, что рангом матрицы называется наибольший размер квадратной подматрицы, определитель которой не равен нулю. При этом определитель - это линейная комбинация с коэффициентами или 1 , или -1 всех чисел, каждое из которых есть произведение всех элементов некоторой диагонали этой подматрицы. Согласно определению 2, граничным рангом матрицы называется наибольший размер квадратной подматрицы, имеющей ненулевой перманент. При этом перманент - это сумма всех чисел, каждое из которых есть произведение всех элементов некоторой диагонали подматрицы. Таким образом, отличие определений ранга и граничного ранга в том, что в одном случае проверяется на равенство нулю некоторая линейная комбинация чисел, а во втором проверяется на равенство нулю сумма тех же чисел.

Докажем неравенство

$$
\rho(A) \geqslant \max _{d \in N_{s}^{k}} \operatorname{rank}\left[B_{A}(d)\right]
$$

Для произвольных матриц $F$ и $G$ из $\mathscr{R}_{m, n}^{+}$, носители которых совпадают, справедливо неравенство

$$
\rho(F) \geqslant \operatorname{rank}(G) .
$$

Так как носители матриц $A$ и $B_{A}(d)$ совпадают для любого $d \in N_{s}^{k}$, для любого указанного $d$ верно неравенство

$$
\rho(A) \geqslant \operatorname{rank}\left[B_{A}(d)\right]
$$

Отсюда следует справедливость неравенства (3). 
Докажем обратное неравенство

$$
\rho(A) \leqslant \max _{d \in N_{s}^{k}} \operatorname{rank}\left[B_{A}(d)\right] .
$$

Пусть $\rho(A)=l$. Из определения граничного ранга следует, что существует подматрица $H$ матрицы $A$ размера $l \times l$ с ненулевым перманентом. Обозначим число ненулевых элементов матрицы $H$ через $i$. Существует вектор $d^{*}$ длины $i$ с дейсгвительными элементами такой, что $\operatorname{det} B_{H}\left(d^{*}\right) \neq 0$. Действительно, определитель матрицы $B_{H}(x)$ является многочленом от $i$ переменных. Так как $\operatorname{per} H \neq 0$, многочлен $\operatorname{det} B_{H}(x)$ не нулевой и представляет собой линейную комбинацию мономов длины $l$. Следовательно, существует вектор $d^{*}$ с действительными элементами такой, что $\operatorname{det} B_{H}\left(d^{*}\right) \neq 0$. Тогда, по лемме 1 , существует вектор $d$ из $N_{s}^{i}, s>1$, такой, что $\operatorname{det} B_{H}(d) \neq 0$.

Выберем вектор $f$ из $N_{s}^{k}$ такой, чтобы на соответствующих местах были расположены компоненты вектора $d$. Тогда очевидно, что

$$
\operatorname{rank}\left[B_{A}(f)\right] \geqslant l .
$$

Следовательно,

$$
l \leqslant \max _{d \in N_{s}^{k}} \operatorname{rank}\left[B_{A}(d)\right] .
$$

Таким образом, доказано неравенство, обратное (3), а значит, установлена справедливость равенства (2).

Лемма доказана.

Доказательство предложения 1. Из леммы 3 следует, что граничный ранг матрицы равен максимуму ранга сопутствующей матрицы по всем векторам из $N_{s}^{k}$. В Алгоритме находится максимум ранга соответствуюцей матрицы только по $l$ векторам из $N_{s}^{k}$. Из этого, в частности, вытекает, что $\rho^{\prime}(A) \leqslant \rho(A)$.

Если $\max _{d \in N_{s}^{k}} \operatorname{rank}\left[B_{A}(d)\right]$ равен $l$, то существует вектор $d$ из $N_{s}^{k}$ и подматрица $H(d)$ матрицы $B_{A}(d)$ такие, что $\operatorname{det} H(d) \neq 0$. Пусть матрица $H(d)$ содержит $v$ ненулевых элементов. Пусть для какого-либо вектора $d(i), i=1, \ldots, l$, из Алгоритма определитель матрицы $H(d(i))$ не равен нулю. Тогда $\operatorname{rank} B_{A}(d(i)) \geqslant l$, и следовательно, $\rho^{\prime}(A) \geqslant \rho(A)$. С учетом неравенства из предыдущего абзаца получаем, что $\rho^{\prime}(A)=\rho(A)$.

Следовательно, вероятность того, что Алгоритм неправильно находит значение $\rho(A)$ (то есть $\rho^{\prime}(A) \neq \rho(A)$ ) можно сверху оценить вероятностью того, что для любого $i=1, \ldots, l$ определитель матрицы $H(d(i))$ равен нулю. С учетом леммы 2, последнюю вероятность можно оценить сверху числом $\left(2 s^{v-1} / s^{v}\right)^{l}=(2 / s)^{l}$.

Предложение 1 доказано.

Замечание 2. Из доказательства предложения 1 следует, что, чем меньше оценка числа $X_{A}$ корней, тем меньше ошибка Алгоритма для фиксированных параметров $s$ и $l$. Экспериментальные данные показывают, что Алгоритм работает лучше, чем это указано в формулировке предложения 1. Другими словами, на практике вероятность неправильного нахождения Алгоритмом граничного ранга меныше, чем это указано в предложении 1.

Доказательство предложения 2. Это утверждение очевидно. Действительно, сложность Алгоритма равна, фактически, сложности нахождения ранга матрицы, умноженной на $l$. В данном случае для нахождения ранга применяется прямой ход алгоритма Гаусса, сложность которого есть $O\left(\mathrm{~nm}^{2}\right)$. 
Замечание 3. При оценке сложности Алгоритма считалось, что параметры $s$ и $l$ имеют фиксированные значения, а к бесконечности стремятся только значения параметров $n$ и $m$. Соответственно, в оценке сложности указан только порядок стремления оценки сверху к бесконечности и не говорится о зависимости от этой оценки $s$ и $l$.

Ясно, что алгоритм Гаусса не является оптимальным алгоритмом для нахождения ранга матрицы. Применение более быстрых алгоритмов позволит улучшить оценку сложности Алгоритма. Например, в [4] указан алгоритм нахождения ранга матрицы, сложность которого есть $O\left(\mathrm{~nm}^{\log _{2} 7-1}\right)$. Учитывая сказанное выше, такой же (по порядку величины) будет и сложность соответствующего Алгоритма для вычисления граничного ранга матрицы.

\section{Список литературы}

1. Сачков В. Н., Тараканов В. Е., Комбинаторика неотрицательных матрич. ТВП, Москва, 2000.

2. Минк Х., Перманенты. Мир, Москва, 1982.

3. Фаддеев Д. К., Фаддеева В. Н., Вычислительные методы линейной алгебры. Физматгиз, Москва, 1960.

4. Солодовников В. И., Верхние оценки сложности решения систем линейных уравнений. Записки научных семинаров Ленинградского ото. Матем. ин-та им. В. А. Стеклова (1982) 118, 159-187.

Статья поступила 11.03.2004. 